\title{
Comparison of C-doped AlN/GaN HEMTs and AlN/GaN/AlGaN double heterostructure for $\mathrm{mmW}$ applications
}

\author{
R. Kabouche ${ }^{1}$, J. Derluyn², R. Püsche ${ }^{2}$, S. Degroote ${ }^{2}$, M. Germain ${ }^{2}$, R. Pecheux ${ }^{1}$, E. Okada ${ }^{1}$, M. Zegaoui ${ }^{1}$, and F. \\ Medjdoub $^{1}$ \\ ${ }^{1}$ IEMN - CNRS, Institute of Electronics, Microelectronics and Nanotechnology, UMR8520 \\ Av. Poincaré, 59650 Villeneuve d'Ascq, France \\ ${ }^{2}$ EpiGaN, Kempische Steenweg 293, 3500 Hasselt, Belgique. \\ riad.kabouche@ed.univ-lille1.fr; farid.medjdoub@iemn.univ-lille1.fr
}

\begin{abstract}
We report on a comparison of the ultrathin (sub$10 \mathrm{~nm}$ barrier thickness) AIN/GaN heterostructure using two types of buffer layers for millimeter-wave applications: 1) carbon doped GaN high electron mobility transistors (HEMTs) and 2) double heterostructure field effect transistor (DHFET). It is observed that the carbon doped HEMT structure shows superior electrical characteristics, with a maximum drain current density $I_{d}$ of $1.5 \mathrm{~A} / \mathrm{mm}$, an extrinsic transconductance $G_{m}$ of $500 \mathrm{mS} / \mathrm{mm}$ and a maximum oscillation frequency $f_{\max }$ of $242 \mathrm{GHz}$ while using a gate length of $120 \mathrm{~nm}$. The C-doped structure delivering high frequency performance together with an excellent electron confinement under high bias enabled to achieve a state-of-the-art combination at $40 \mathrm{GHz}$ of output power density (Pout $=7$ $\mathrm{W} / \mathrm{mm}$ ) and power added efficiency (PAE) above $52 \%$ up to $V_{D S}$ $=25 \mathrm{~V}$ in pulsed mode.
\end{abstract}

\section{INTRODUCTION}

With the development of wireless communication such as $5 \mathrm{G}$ or SATCOM, the need and requirements for millimeterwave compact solid-state high power amplification has significantly increased. Achieving high power-addedefficiency and output-power-density combination in the millimeter-wave range represents currently one of the key goal for the GaN technology. Indeed, higher PAE not only saves electrical power usage but also can reduce the size and cost of high power amplifiers (HPAs), due to the lower amount of heat dissipated. For instance, in space applications, the traveling wave tube amplifiers (TWTA) are still widely used, because of the high PAE while delivering high Pout. Even though attractive efficiencies on $\mathrm{GaN}$ devices (well-beyond $40 \%$ ) have been already demonstrated up to $\mathrm{Ka}$ band $[1][2][3][4][5]$, rather limited PAE has been reported so far in the $\mathrm{Q}$ band $(40 \mathrm{GHz})$ and above. For high frequency applications requiring short gate lengths, it has been shown that the double heterostructure using a thick AlGaN back barrier with $8 \%$ Aluminum $(\mathrm{Al})$ content enabled to combine a high electron confinement with high frequency performance and low trapping effects [6]. It can be noticed that the record frequency performances achieved on devices fabricated by HRL laboratory employed an AlGaN back barrier [7] as well. However, AlGaN alloys have the drawback that they have much poorer thermal conductivity than the binary GaN or AlN alloys[8], which causes an increase in the peak channel temperature during operation. C-doped GaN buffers have been widely adopted in high voltage power switching applications[9], and have the advantage compared to Fe-doped GaN buffers of lower memory effects[10], lower risk of diffusion and the elimination of contamination risks when used in Si CMOS-based foundries..

In this paper, the two types of buffer layer structure, DHFET and C-doped HEMT are evaluated and compared using a 0.12 $\mu \mathrm{m}$ gate technology.

\section{MATERIAL AND D EVICE PROCESSING}

The AlN/GaN heterostructures were grown by metal organic chemical vapor deposition (MOCVD) on 4 in. $\mathrm{SiC}$ substrates. The HEMT structure consists of transition layers to GaN, a $1 \mu$ m-thick C-doped GaN buffer layer followed by an undoped GaN channel, a $4.0 \mathrm{~nm}$ ultrathin AlN barrier layer and a 10-nm-thick in situ $\mathrm{Si}_{3} \mathrm{~N}_{4}$ cap layer (Fig. 2.1). The in situ $\mathrm{SiN}$ layer is used both as early passivation as well as to prevent strain relaxation [11][12][13]. In the second structure called DHFET, the 1- $\mu$ m-thick C-doped GaN buffer layer is replaced with a $1 \mu \mathrm{m}$-thick $\mathrm{Al}_{0.08} \mathrm{Ga}_{0.92} \mathrm{~N}$ layer and a $150 \mathrm{~nm}$ GaN channel as shown in Fig. 2.1. Room-temperature Hall measurements showed high electron sheet concentrations of $1.8 \times 10^{13}$ and $1.6 \times 10^{13} \mathrm{~cm}^{-2}$ with an electron mobility about $1100 \mathrm{~cm}^{2} \mathrm{~V}^{-1} \mathrm{~s}^{-1}$ in the HEMT and DHFET heterostructures, respectively. a)

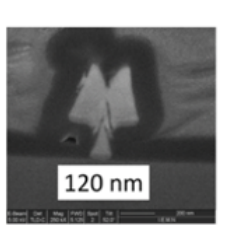

b)

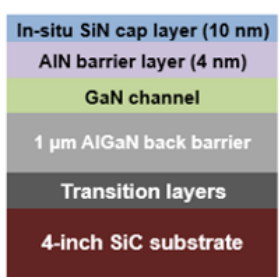

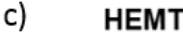

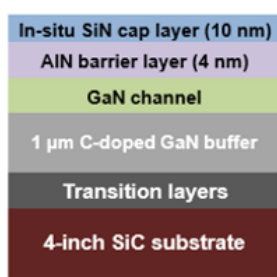

Fig. 2.1. : a) FIB view of the $0.12 \mu \mathrm{m}$ T-gate and schematic cross section of b) DHFET and c) C-doped HEMT.

A $\mathrm{Ti} / \mathrm{Al} / \mathrm{Ni} / \mathrm{Au}$ metal stack followed by a rapid thermal annealed has been used to form the ohmic contacts directly on top of the AlN barrier layer by etching the in situ $\mathrm{Si}_{3} \mathrm{~N}_{4}$ layer. Device isolation was achieved by nitrogen implantation. 
Ohmic contact resistance $\left(\mathrm{R}_{\mathrm{c}}\right)$ extracted from linear transmission line model (TLM) structures was as low as 0.3 $\Omega . \mathrm{mm}$ for both heterostructures. Then, a $0.12 \mu \mathrm{m} \mathrm{Ni} / \mathrm{Au} \mathrm{T}-$ gate length was defined by e-beam lithography (see Fig. 2.1). The $\mathrm{SiN}$ underneath the gate was fully removed by $\mathrm{SF}_{6}$ plasma etching. The gate-source and gate-drain spacings were 0.3 and $2 \mu \mathrm{m}$, respectively, and the device width was $50 \mu \mathrm{m}$. Finally, $200 \mathrm{~nm} \mathrm{PECVD} \mathrm{Si}_{3} \mathrm{~N}_{4}$ was deposited as final passivation.

\section{DC AND SMALL SIGNAL CHARACTERIZATION}

DC measurements have been performed with a Keysight A2902A static modular and source monitor. The Fig 3.1 shows the typical I-V characteristics of both structures. The gate source voltage was swept from $-6 \mathrm{~V}$ to $+2 \mathrm{~V}$ with a step of $0.5 \mathrm{~V}$. For the DHFET, a maximum current drain density ( $\mathrm{I}_{\mathrm{Dmax}}$ ) of $1.3 \mathrm{~A} / \mathrm{mm}$ is observed at $\mathrm{V}_{\mathrm{DS}}=10 \mathrm{~V}$. For the HEMT, a higher $I_{D \max }$ of $1.5 \mathrm{~A} / \mathrm{mm}$ is obtained under the same conditions reflecting the higher carrier concentration.
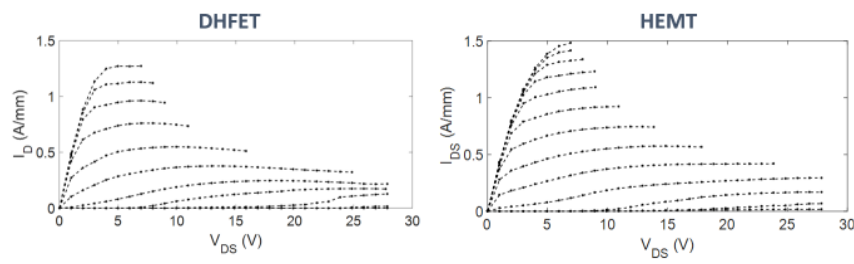

Fig. 3.1. : Output characteristics of DHFET and C-doped HEMT.

The transfer characteristics of both structures at $\mathrm{V}_{\mathrm{DS}}=6,8$ and $10 \mathrm{~V}$ are shown in Fig. 3.2. Excellent device pinch-off behavior are obtained in both cases with a low off-state leakage current well-below $10 \mu \mathrm{A} / \mathrm{mm}$. This confirms that a good electron confinement can be also obtained without the use of an AlGaN back barrier, even when using short gate lengths. At the same time, a slightly higher extrinsic transconductance is observed for the HEMT structure with a $\mathrm{G}_{\mathrm{m}}$ around 500 $\mathrm{mS} / \mathrm{mm}$ at $\mathrm{V}_{\mathrm{DS}}=10 \mathrm{~V}$ against $470 \mathrm{mS} / \mathrm{mm}$ at $\mathrm{V}_{\mathrm{DS}}=10 \mathrm{~V}$ for the DHFET. At these respective biases, the cut-off frequency and maximum frequency oscillation are extracted from the scattering (S) parameters using Rhode and Schwarz ZVA67GHz. The DHFET yields a $\mathrm{f}_{\mathrm{T}}=56 \mathrm{GHz}$ and $\mathrm{f}_{\max }=235$ $\mathrm{GHz}$ while rather similar RF performances are achieved for the HEMT with $\mathrm{f}_{\mathrm{T}}=60 \mathrm{GHz}$ and $\mathrm{f}_{\max }=242 \mathrm{GHz}$ (see Fig. 3.3). It can be stressed that a high $\mathrm{f}_{\max } / \mathrm{f}_{\mathrm{T}}$ ratio above 4 is observed, which is quite untypical. This is due to the favorable aspect ratio between the gate length and the gate to channel distance (ultrathin $4 \mathrm{~nm}$ barrier), the high carrier concentration and the low parasitic gate capacitance.
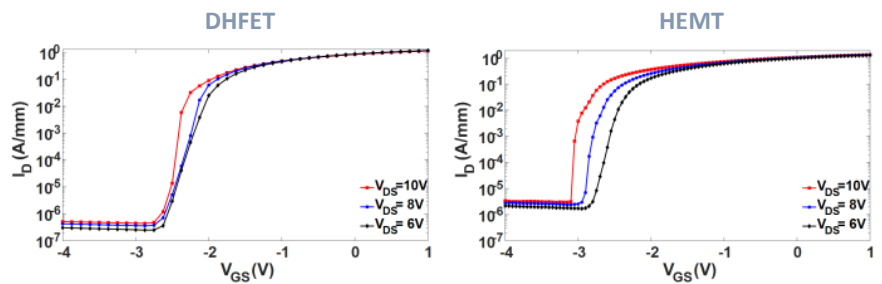

Fig. 3.2. : Transfer characteristics at $\mathrm{V}_{\mathrm{DS}}=6,8,10 \mathrm{~V}$ of DHFET and HEMT.

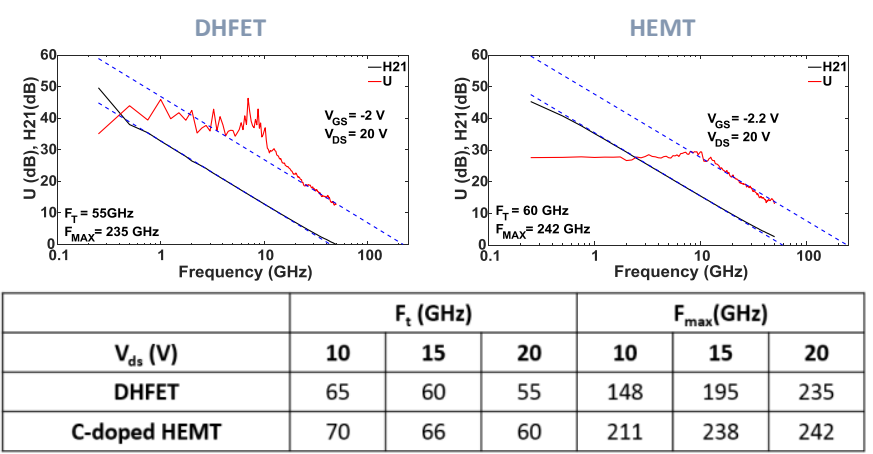

Fig. 3.3. : RF performance of the $0.12 \mu \mathrm{m}$ of DHFET and C-doped HEMT.

\section{LARGE SIGNAL CHARACTERIZATION AT $40 \mathrm{GHZ}$}

Large signal characterizations at $40 \mathrm{GHz}$ have been carried out on both structures in pulsed mode $(1 \mu \mathrm{s}$ width and $1 \%$ duty cycle). It can be stressed that the same measurement conditions (deep AB class) have been used for the HEMTs and DHFETs. Fig 4.1 shows the pulsed power performance of a $0.12 \times 50 \mu \mathrm{m}^{2}$ AlN/GaN HEMT at $40 \mathrm{GHz}$ with $\mathrm{V}_{\mathrm{DS}}=15 \mathrm{~V}, 20 \mathrm{~V}$, and $25 \mathrm{~V}$. A saturated Pout (output power density) of $7 \mathrm{~W} / \mathrm{mm}$ was achieved with a peak PAE of $52 \%$ associated to a linear power gain above $8 \mathrm{~dB}$. Moreover, at $\mathrm{V}_{\mathrm{DS}}=10 \mathrm{~V}$ a peak $\mathrm{PAE}$ as high as $56 \%$ combined with an output power density of $1.6 \mathrm{~W} / \mathrm{mm}$ has been reached.

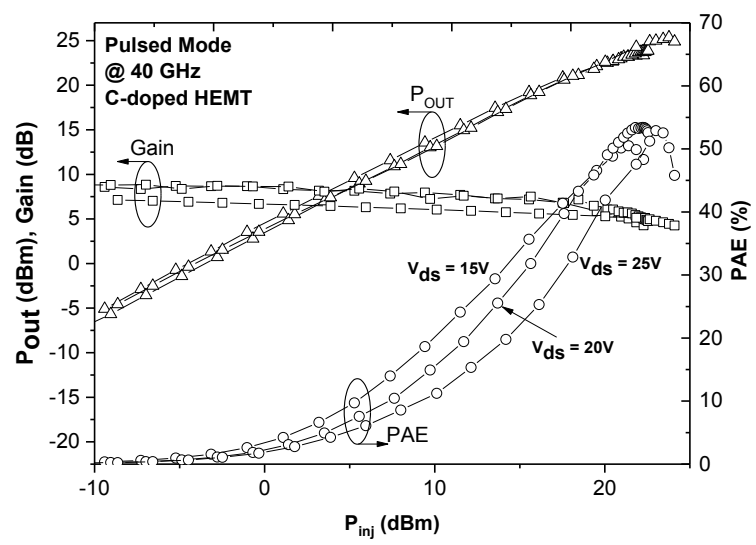

Fig. 4.1. : Pulsed power performance of a $0.12 \times 50 \mu \mathrm{m}^{2} \mathrm{HEMT}$ at $40 \mathrm{GHz}$ with $\mathrm{V}_{\mathrm{DS}}=15,20,25 \mathrm{~V}$.

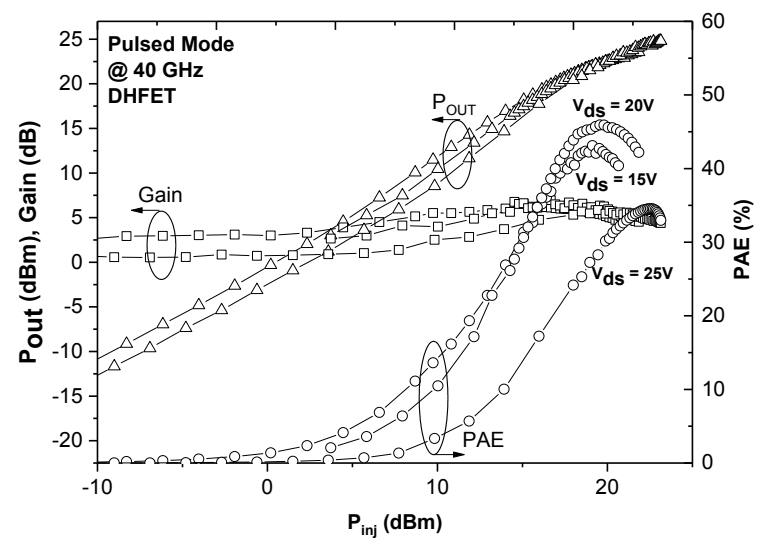

Fig. 4.2. : Pulsed power performance of a $0.12 \times 50 \mu \mathrm{m}^{2}$ DHFET at $40 \mathrm{GHz}$ with $\mathrm{V}_{\mathrm{DS}}=15,20,25 \mathrm{~V}$. 
Similarly the pulsed power performance of a $0.12 \times 50$ the DHFET structure at $40 \mathrm{GHz}$ can be seen in Fig. 4.2. The output power density evolves linearly as a function of the drain bias (see Fig 4.3) with no sign of saturation even at $\mathrm{V}_{\mathrm{DS}}=25 \mathrm{~V}$ for both structure reflecting the high material quality and associated processing. It is worth noting that the C-doped structure shows the ability to deliver a PAE above $50 \%$ up to $\mathrm{V}_{\mathrm{DS}}=25 \mathrm{~V}$. For the DHFET, the PAE shows an increasing evolution up to $\mathrm{V}_{\mathrm{DS}}=20 \mathrm{~V}$. At $\mathrm{V}_{\mathrm{DS}}=15 \mathrm{~V}$, a PAE of $43 \%$ is obtained while a PAE of $46 \%$ associated to an output power density of $5.1 \mathrm{~W} / \mathrm{mm}$ at $\mathrm{V}_{\mathrm{DS}}=20 \mathrm{~V}$. Nevertheless, at $\mathrm{V}_{\mathrm{DS}}$ $=25 \mathrm{~V}$ a degradation of performance is observed with a PAE decreasing to $35 \%$. The significant drop of the PAE at $\mathrm{V}_{\mathrm{DS}}=25 \mathrm{~V}$ can be explain by the fact that, despite the pulsed mode, the $1 \mu \mathrm{s}$ pulse width is still too large to avoid the selfheating at such a high voltage within the DHFET structure.

Consequently, the performance enhancement is attributed to the better thermal dissipation within the C-doped GaN HEMTs as compared the DHFETs. It can be noticed that no device degradation is observed in both cases as seen for instance from the absence of gate leakage current increase subsequent to the number of pulsed power sweeps up to $\mathrm{V}_{\mathrm{DS}}=25 \mathrm{~V}$.

As can be seen from the benchmark in Fig. 4.4, the achieved PAE / P PUT combination at $40 \mathrm{GHz}$ compares favorably to the state-of-the-art.

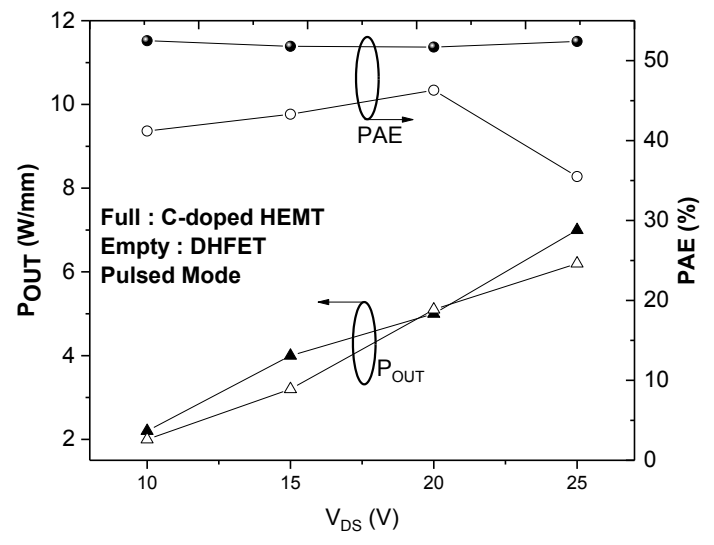

Fig. 4.3. : Pulsed output power density (triangle) and PAE (circle) versus $\mathrm{V}_{\mathrm{DS}}$ at $40 \mathrm{GHz}$.

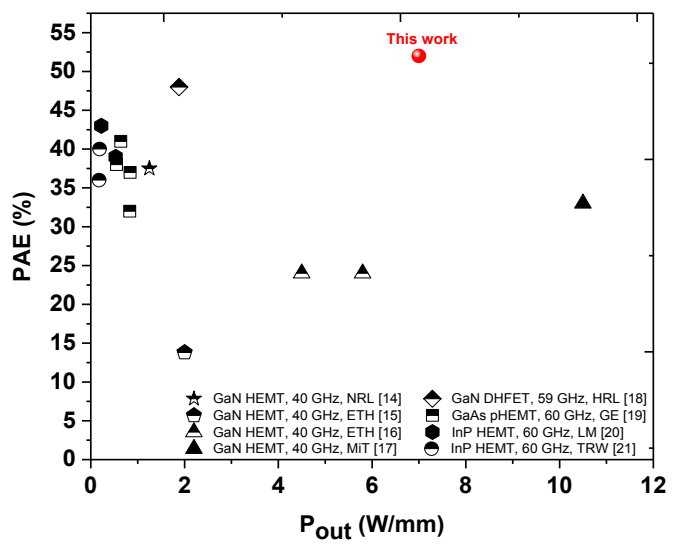

Fig. 4.4. : Benchmark of the output RF power density vs PAE for Q and $\mathrm{V}$ band[14][15][16][17][18][19] [20][21].

\section{CONCLUSION}

This work shows that a careful architecture of buffer layers should be employed in order to perform high performance millimeter-wave GaN devices. The use of higher bias operation $\left(\mathrm{V}_{\mathrm{DS}} \geq 20 \mathrm{~V}\right)$ will be possible while using short gate length only if the thermal resistance induced by the buffer layers is reduced. In particular, it is shown that a thick AlGaN back barrier (DHFET structure) results in a huge drop of PAE at $\mathrm{V}_{\mathrm{DS}}=25 \mathrm{~V}$ despite the pulsed mode. With an enhanced thermal dissipation as compared to the DHFET, the C-doped structure delivers much higher performances illustrated by a state-of-the-art combination of PAE $(>50 \%)$ and an output power density of $7 \mathrm{~W} / \mathrm{mm}$ at $40 \mathrm{GHz}$ and $\mathrm{V}_{\mathrm{DS}}=25 \mathrm{~V}$.

\section{ACKNOWLEDGMENT}

This work was supported by the French RENATECH network and the French Defense Procurement Agency (DGA) under the project EDA-EuGaNiC and contract FUI-VeGaN.

\section{REFERENCES}

[1] P. Saad, H. M. Nemati, M. Thorsell, K. Andersson, and C. Fager, "An inverse class-F GaN HEMT power amplifier with 78\% PAE at $3.5 \mathrm{GHz}$," Eur. Microw. Week 2009, EuMW 2009 Sci. Prog. Qual. Radiofreq. Conf. Proc. - 39th Eur. Microw. Conf. EuMC 2009, no. October, pp. 496-499, 2009.

[2] J. S. Moon et al., " $>70 \%$ power-added-efficiency dual-gate, cascode GaN HEMTs without harmonic tuning," IEEE Electron Device Lett., vol. 37, no. 3, pp. 272-275, 2016.

[3] K. Takagi, S. Takatsuka, Y. Kashiwabara, and S. Teramoto, "Ku-Band AlGaN / GaN-HEMT with over $30 \%$ of PAE," Engineering, pp. 457-460, 2009.

[4] J. S. Moon et al., "55\% PAE and high power Ka-band GaN HEMTs with linearized transconductance via $\mathrm{n}+$ GaN source contact ledge," IEEE Electron Device Lett., vol. 29, no. 8, pp. 834-837, 2008.

[5] A. Crespo et al., "High-power ka-band performance of AlInN/GaN HEMT with 9.8-nm-thin barrier," IEEE Electron Device Lett., vol. 31, no. 1, pp. 2-4, 2010.

[6] F. Medjdoub, M. Zegaoui, B. Grimbert, N. Rolland, and P.-A. Rolland, "Effects of AlGaN Back Barrier on AlN/GaN-on-Silicon High-Electron-Mobility Transistors," Appl. Phys. Express, vol. 4, no. 12, p. 124101, Nov. 2011.

[7] Y. Tang et al., "Ultrahigh-Speed GaN High-ElectronMobility Transistors With fT/fmax of $454 / 444 \mathrm{GHz}$," IEEE Electron Device Lett., vol. 36, no. 6, pp. 549$551,2015$.

[8] W. Liu and A. A. Balandin, "Thermal conduction in AlxGa1-xN alloys and thin films," J. Appl. Phys., vol. 97, no. 7, pp. 1-6, 2005. 
[9] P. Moens, A. Banerjee, P. Coppens, F. Declercq, and M. Tack, "AlGaN / GaN Power Device Technology for High Current ( $100+$ A ) and High Voltage ( 1.2 kV )," Proc. 2016 28th ISPSD, Prague, Czech Repub., no. 1 , pp. $455-458$.

[10] M. J. Uren, J. Moreke, and M. Kuball, "Buffer design to minimize current collapse in GaN/AlGaN HFETs," IEEE Trans. Electron Devices, vol. 59, no. 12, pp. 3327-3333, 2012.

[11] J. Derluyn et al., "Improvement of AlGaN/GaN high electron mobility transistor structures by in situ deposition of a Si3N4 surface layer," J. Appl. Phys., vol. 98, no. 5, p. 54501, 2005.

[12] D. Marcon et al., "Excellent Stability of GaN-on-Si High Electron Mobility Transistors with $5 \mu \mathrm{m}$ GateDrain Spacing Tested in Off-State at a Record Drain Voltage of $200 \mathrm{~V}$ and $200{ }^{\circ} \mathrm{C}$," Jpn. J. Appl. Phys., vol. 49, no. 4, p. 04DF07, Apr. 2010.

[13] D. Marcon et al., "High temperature on- and off-state stress of GaN-on-Si HEMTs with in-situ Si3N4cap layer," IEEE Int. Reliab. Phys. Symp. Proc., pp. 146$151,2010$.

[14] B. P. Downey, D. J. Meyer, D. S. Katzer, and J. A. Roussos, "SiNx /InAlN/AlN/GaN MIS-HEMTs With $10.8 \mathrm{THz} \cdot \mathrm{V}$ Johnson Figure of Merit," IEEE Electron Device Lett., vol. 35, no. 5, pp. 527-529, 2014.

[15] D. Marti, S. Tirelli, A. R. Alt, J. Roberts, and C. R. Bolognesi, "150-GHz cutoff frequencies and 2-W/mm output power at $40 \mathrm{GHz}$ in a millimeter-wave $\mathrm{AlGaN} / \mathrm{GaN}$ HEMT technology on silicon," IEEE Electron Device Lett., vol. 33, no. 10, pp. 1372-1374, 2012.

[16] S. Tirelli, L. Lugani, D. Marti, J. F. Carlin, N. Grandjean, and C. R. Bolognesi, "AlInN-based HEMTs for large-signal operation at $40 \mathrm{GHz}$," IEEE Trans. Electron Devices, vol. 60, no. 10, pp. 30913098, 2013.

[17] T. Palacios et al., "High-power AlGaN/GaN HEMTs for Ka-band applications," IEEE Electron Device Lett., vol. 26, no. 11, pp. 781-783, 2005.

[18] M. Micovic et al., "GaN DHFETs Having 48\% Power Added Efficiency and 57\% Drain Efficiency at Vband," IEEE Electron Device Lett., vol. 38, no. 12, pp. $1-1,2017$.

[19] MING-YIH KAO et al., "Very High Power-Added Efficiency and Low-Noise," vol. I, no. 12, pp. 580$582,1989$.

[20] W. M. T. Kong et al., "Very high efficiency V-band power InP HEMT MMICs," IEEE Electron Device Lett., vol. 21, no. 11, pp. 521-523, 2000.

[21] R. Grundbacher et al., "Pseudomorphic InP HEMT's with dry-etched source vias having $190 \mathrm{~mW}$ output power and 40\% PAE at V-band," IEEE Electron Device Lett., vol. 20, no. 10, pp. 517-519, 1999. 